\title{
CONSIDERACIÓN DE LOS INTANGIBLES EN LA EVALUACIÓN DE ACCIONES POR LOS ANALISTAS FINANCIEROS
}

\author{
Elena Valenzuela D.*
}

Facultad de Ciencias Económicas y Administrativas, Universidad de Chile

(Recibido 2 de febrero 2004, aceptado 20 de mayo 2004)

\section{Resumen}

Tradicionalmente, la información financiera ha dominado las decisiones de inversión y el análisis de activos intangibles ha tomado creciente importancia. El objetivo de este estudio es establecer la relevancia de los intangibles que asignan los analistas financieros al estudiar las empresas y recomendar sus acciones. Se señalan los factores más relevantes que guían esa decisión y su ponderación en empresas de distintos sectores económicos. Se encuestó al $76.1 \%$ de los corredores de bolsa ubicados en Santiago de Chile. Los resultados evidencian el empleo de información no financiera en la recomendación de compra de acciones, reconociendo que varía según el sector económico.

\section{Abstract}

Traditionally, financial information has dominated investment decisions and analysis of intangible assets has taken increasing importance. The objective of this study is to establish the relevance of the intangible assets that financial analysts assign in studying the firms and recommending actions. We indicate the more relevant factors that lead such a decision and its weighs in firms of different economic sectors. We carry out a survey of $76.1 \%$ of local stockbrokers in Santiago de Chile. The results demonstrate evidence of the use of non financial information in the recommendation of the purchase of actions, recognizing that results may vary according to the economic sector.

Clasificación JEL: G14, M40

Palabras clave: Información y mercados, Análisis financiero

* Departamento de Administración, Facultad de Ciencias Económicas y Administrativas Universidad de Chile. Diagonal Paraguay No. 257, Santiago de Chile, Of, 1101. Telèıno: 6783703 y 366. Correo electrónico: evalenz@negocios.uchile.cl 


\section{Introducción}

Una serie de fuerzas influyentes están redefiniendo la economía y la manera de entender los negocios. Estas fuerzas del cambio incluyen, entre otras: el fenómeno de la globalización, los cada vez más elevados niveles de competitividad y complejidad del entorno, las nuevas tecnologías, la mayor frecuencia con que se producen los cambios de demanda de los clientes, así como también la disminución del ciclo de vida de los productos.

Estamos inmersos en un sistema imparable hacia lo que viene denominándose "Era del Conocimiento" o "Sociedad del Conocimiento", donde la tecnología sigue siendo una palanca fundamental, pero cada vez menos diferenciadora; es el conocimiento y la gestión de ese conocimiento el que permitirá a las empresas adaptarse mejor y más rápidamente a los cambios.

Tradicionalmente, los activos tangibles (capital físico y financiero) constituían el activo más preciado de las empresas. Sin embargo, en los últimos años esta idea ha dado paso a la consideración cada vez más creciente de la categoría de activos intangibles o capital intelectual, ${ }^{1}$ como clave para competir en entornos dinámicos y complejos.

Algunos autores (Amir y Lev (1996)), han examinado la fluctuación de la razón valor de mercado a valor libro, encontrando que existe un gran desfase entre ambos valores en los últimos años. Esta diferencia puede estar dada por la existencia de activos intangibles que no son incorporados en el valor libro de la empresa, pero que si son valorados por el mercado. Esto se debe en parte, al papel conservador de la contabilidad establecido en los "principios generalmente aceptados", nos referimos principalmente al principio de "costo histórico" 2 y "criterio prudencial". ${ }^{3}$ Asimismo la definición de "activos", como "Recursos que tienen el potencial de generar a la firma, beneficios económicos futuros, que puedan ser medidos o cuantificados" (Sidney et al. (1982, pp. 35)), deja en claro la razón de que los activos intangibles son contabilizados generalmente como gastos del período y reflejados en el Estado de Resultados, ya que no existe certeza de que generen beneficios futuros cuantificables.

El hecho de que las inversiones en activos intangibles sean consideradas, como gastos contables del período, podría explicar la existencia de correlaciones negativas entre estas inversiones y la rentabilidad contable de la empresa, generando una reducción del valor de la empresa, cuando el criterio de valoración se basa en la rentabilidad financiera o económica.

En estas condiciones, la contabilidad pasa a ser cada vez menos importante para valorar las empresas, ya que sólo otorga información del pasado, aún

1 Hay muchas definiciones de capital intelectual, pero según el Instituto Euroforum Escorial, (Euroforum (1998, pp. 21)) indica que en general el término Capital Intelectual se emplea como "Conjunto de Activos de una sociedad que, pese a no estar reflejados en los estados contables tradicionales, generan o generarán valor para la enpresa en el futuro".

2 Indica que "el registro de las operaciones se basa en costos históricos..." (Boletín Técnico No. 1, Colegio de Contadores).

3 La medición de recursos y obligaciones en la contabilidad requiere que estimaciones sean incorporadas y frente a alternativas para esas estimaciones debe elegirse la más conservadora.... (Boletín Técnico No. 1, Colegio de Contadores). 
cuando sigue siendo valiosa para conocer los resultados financieros de la empresa y para efectos de control y gestión. Sin embargo, se hace necesario complementar los estados financieros con informes de los recursos intangibles que posee cada empresa tal como lo han hecho algunas compañías en el mundo, como por ejemplo Skandia y Celemi (Serrano (2000)), que demuestran la utilidad de dichos informes al comunicarlo a los accionistas y potenciales inversionistas, y mostrar la fuente de sus ventajas competitivas que le permiten la creación de valor. Por ello, vemos que las empresas están en constante mejora de su gestión y calidad de la administración, y abocados explícitamente a establecer ventajas competitivas a través de su capital intelectual, tratando de crear valor.

Identificar estos activos intangibles y estructurarlos, son procesos necesarios para poder gestionarlos y, tal como dice Ross (1998, pp.1) refiriéndose al capital intelectual: "Hay que ser capaz de capturarlo, medirlo y evaluarlo porque es la fuente que generará las futuras ganancias de las empresas".

En otros países, como se verá en la versión bibliográfica, la literatura ha enfatizado la creciente importancia de la revelación de los intangibles por parte de las empresas, debido a que los agentes del mercado de capitales, es decir, los analistas e inversionistas demandan mayor información de este tipo para hacer sus análisis, y así lograr pronósticos más certeros del valor de la empresa.

El objetivo de este estudio es conocer la consideración de los activos intangibles, que hacen los analistas financieros en sus estudios para valorar una empresa y hacer sus recomendaciones. Específicamente se desea investigar si se han incorporado en sus estudios, las actividades y activos intangibles (variables no financieras) que poseen las empresas; establecer cuáles son esas variables, las ponderaciones que se le asignan a las empresas en los diferentes sectores económicos; como asimismo, conocer las mediciones y fuentes de información que se emplean.

En la sección 2 de este artículo se hace una revisión bibliográfica; posteriormente en la sección 3 se describe el estudio empírico, señalando la metodología y los resultados de él. Finalmente, en la sección 4 se indican las conclusiones.

\section{Revisión bibliográfica}

En los últimos años, la importancia del capital intelectual ha sido enfatizada en la literatura y muchos estudios han mostrado las limitaciones de la contabilidad tradicional y la creciente necesidad de contar con datos no financieros. Sullivan y Sullivan (2000) establecen que los métodos tradicionales de contabilidad son inadecuados para valorar compañías cuyos activos son en su mayoría intangibles; Gelb (2002) encuentra que firmas con altos niveles de activos intangibles, perciben que la contabilidad es inefectiva en comunicar y otorgar información a los inversıonistas y que se requiere de información adicional que sea publicada voluntarianıente por las firmas. Recientes estudios han demostrado el deterioro de la relación estadística entre las variables financieras, tales como ingresos, valores libro, flujo de caja y el precio de las acciones (Lev y Zarowin (1999); Brown et al. (1999)). Sin embargo es importante recalcar que esto no implica que los resultados financieros sean irrelevantes, más bien son muy importantes, pero ell menor grado cuando ella no va a acompañada de información complementaria.

Se ha tomado conciencia de la importancia de entregar información no financiera por parte de las empresas y, estudios recientes hacen un llamado a 
mejorar la revelación de los intangibles por parte de las empresas y a desarrollar nuevos modelos de información y reportes sobre capital intelectual o activos intangibles (Meritum (2002)).

La creciente necesidad del mercado de capitales de poder obtener una mayor revelación de información adicional, está relacionada con un creciente interés en la transparencia de los informes de las empresas y de la comunicación de los impulsores de la creación de valor de las compañías. En consecuencia, un gran número de organizaciones, especialmente nórdicas, han comenzado a publicar voluntariamente su capital intelectual en los últimos años, para que de esta forma, se puedan visualizar sus esfuerzos y recursos en relación a los empleados, consumidores, procesos y tecnología y, además, cumplir con la necesidad del mercado de brindar una mayor transparencia (Bukh, Nielsen, Gormsen y Mouritsen (2003)).

A diferencia de los informes financieros, los informes de capital intelectual no tienen un conjunto de estándares contables. Sin embargo en los últimos años, algunos informes en Dinamarca han mostrado que es posible construir un modelo para informar sobre las fuentes intelectuales y competencias de la compañía. En la práctica, un informe de capital intelectual puede ser revelado en forma escrita, por medio de dibujos y por ilustraciones, para presentar los esfuerzos de las organizaciones en la administración del conocimiento (Bukh, Nielsen, Gormsen y Mouritsen (2003)).

Algunos estudios empíricos sobre la información y comunicación de los activos intangibles por parte de las organizaciones, han marcado la diferencia que existe entre las distintas visiones que tienen los analistas, inversionistas y los gerentes de empresas. Eccles junto con otros autores (Eccles y Mavrinac (1995); Eccles et al. (2001)) estudiaron la percepción que tienen estos tres agentes del mercado, sobre la calidad de la información otorgada por las corporaciones en relación a los mercados financieros. Sus resultados indicaron que existe una gran diferencia entre las expectativas de información existentes, entre las compañías y el mercado de capitales, es decir, hay una falta de comunicación entre los gerentes y los inversionistas, sobretodo en las organizaciones intensivas en conocimiento. Los gerentes ven su propia política de comunicación como algo proactivo, mientras que los analistas financieros encuentran que esta información es inadecuada y más reactiva que proactiva.

Por su parte, Gleb y Zarowin (2002, sintetizado en Bukh, Nielsen, Gormsen y Mouritsen (2003 pp. 6)) muestran que la mayor entrega de información beneficia a los inversionistas, pues una mayor revelación está asociada a los precios de las acciones y a los ingresos futuros de las empresas.

Un estudio realizado por Ernest \& Young en Estados Unidos (Blitz et al. (1997)) indicó que los analistas financieros y los inversionistas usan variables no financieras para el análisis de las compañías que estudian, encontrando que los datos no financieros utilizados por cada agente mencionado, dependen del tipo de industria en que se encuentre la empresa; asimismo, se señala que los factores no financieros más considerados son la "ejecución de la estrategia" y la "inmovación". Para el caso de los inversionistas, ese estudio indicó que el $35 \%$ de las decisiones de inversión se guían por consideraciones de información no financiera; un trabajo similar realizado en Inglaterra encontró resultados parecidos (Eales et al. (2000)). 
En la misma línea de los estudios anteriores, Valenzuela (2003) analiza la relación entre los factores no financieros en las decisiones de inversión de los inversionistas institucionales en la economía chilena, encontrando que cerca de un quinto de los inversionistas institucionales que operan en el país asignan entre $81 \%$ y $100 \%$ de importancia a los factores no financieros al momento de evaluar una posible inversión en acciones, los resultados de este estudio también concuerdan con los obtenidos en Estados Unidos (Blitz et al. (1997)) y en Inglaterra (Eales et al. (2000)) al asignar gran importancia a los factores no financieros relacionados con la gestión corporativa, especialmente la "experiencia del grupo directivo" y la "ejecución de la estrategia corporativa".

Garcia-Meca (2003) a través de un análisis de los informes de analistas financieros con mayor volumen de negocio, encuentra que los analistas usan información sobre capital intelectual para apoyar sus recomendaciones de compra o venta de títulos de las compañías que cubren, la autora observa que la información estratégica ha estado presente en todos los informes analizados, seguida de información sobre la clientela $(65.9 \%)$ y procesos $(59.9 \%)$. Casi en la mitad de esos informes, se comenta la relación de los Recursos Humanos con la empresa $(44.2 \%)$, la tecnología empleada $(41.5 \%)$ y, en último lugar, se reconoció la información sobre investigación y desarrollo (30\%); interpretado por la autora por la poca información divulgada por la compañía al respecto y por la dificultad para hacer una estimación precisa y objetiva. A su vez, la autora identifica que para las recomendaciones de compra se usa más intensivamente la inforınación no financiera.

Mavrinac y Boyle (1996; sintetizado en Bukh, Nielsen, Gormsen y Mouritsen (2003)) analizaron los reportes de los analistas financieros para determinar si ellos tomaban en cuenta la información no financiera de las firmas al moinento de valorarlas. Los resultados indicaron que los analistas atribuyen un valor significativo a la información no financiera, especialmente analistas que trabajan con organizaciones intensivas en conocimiento. Los reportes que los analistas hacen de este tipo de organizaciones, demuestran que temas como el entrenamiento o la capacitación de los empleados, aparecen como factores relevautes al momento de estimar el crecimiento potencial futuro de las empresas.

Asimismo, Beattie (1999; sintetizado en Bukh, Nielsen, Gormsen y Mouritsen (2003)) estudió la capacidad que tienen los reportes financieros para satisfacer la demanda de información del mercado de capitales. Los resultados indicaron una creciente atención en los factores no financieros por parte de los analistas o inversionistas, pero con menor peso que los factores financieros. Los encuestados de este estudio expresaron su interés por una mayor información acerca de los factores de riesgo y por información confiable acerca de la calidad y la experiencia de la administración, además de su integridad. Asimismo, este tipo de información es vista como relevante y como un factor determinante del éxito de una organización, en cuanto a su habilidad para crear valor.

Los resultados anteriores están en concordancia con Anderson y Epstein (1996) y con Barlett y Chandler (1997), quienes recalcaron que la comunicación de una organización no debería estar enfatizada sólo en informar a los sofisticados inversionistas financieros, sino que también a aquellos inversionistas y accionistas con menos experiencia.

La empresa PricewaterhouseCoopers se ha destacado por hacer investi- 
gaciones globales para explotar las necesidades de las compañías de otorgar información adicional a los balances y la existencia de una demanda del mercado de un nuevo modelo de reporte, que ellos denominan "Value Reporting". La investigación se realizó en varios países, tales como Inglaterra (Coleman y Eccles (1997)), Estados Unidos (Eccles y Kahn (1998)), Australia (Morris et al. (1998)) y Suiza (Eccles y Weibel (1998)), entre otros. Los resultados de estos estudios mostraron que los analistas tienen una mayor necesidad de información para la toma de decisiones que los inversionistas comunes, por otra parte se encuentra que existen algunas percepciones distintas entre agente financiero e inversionistas acerca de cuales son las medidas relevantes para invertir en una firma en particular, se señaló que la medida más relevante para los analistas en Estados Unidos y Australia son los "ingresos de la firma" primer lugar, y en Inglaterra y Suiza ellos ocupan el segundo lugar. Los "gastos de capital" están en primer lugar en Australia y Suiza.

Las variables no financieras más importantes son el "desempeño de la industria" número uno en Inglaterra y Estados Unidos, el "crecimiento del mercado" y la "participación de mercado".

En Inglaterra también se realizó un estudio a través del Market Opinión \& Research Internacional (MORI) durante el año de 1997 a 100 ejecutivós (Eccles et al. (1998)), donde se comparó la importancia que le dan los ejecutivos a las medidas de resultado de la empresa con el valor que le asignan a ellas los inversionistas y los analistas cuando toman decisiones de inversión. Se demostró que los inversionistas y analistas no recibían la información adecuada, y que ambos agentes piensan que, aún cuando los ejecutivos se preocupan de comunicar la información de la empresa, las compañías hacen un trabajo bastante pobre al momento de comunicar la información financiera y no financiera. Los resultados mostraron también que para los ejecutivos de las empresas, las variables más importantes son las financieras, tales como "flujo de caja" (78\%), "ingresos" (76\%), "costos" (73\%) y "gastos de capital" (71\%), aun cuando las variables no financieras también son consideradas como importantes, pero en menor grado; las de mayor relevancia resultaron ser "satisfacción del cliente" (66\%), "participación de mercado" (65\%) y "crecimiento de mercado" (60\%). Pero el factor cualitativo considerado como el más importante fueron las "metas estratégicas" de la empresa ( $71 \%)$.

El resultado de estos estudios es que, en un mundo con un creciente desarrollo tecnológico, ciclos de vida de productos cada vez más cortos y una creciente integración de los mercados de capitales, el valor de las empresas es reflejado de mejor manera si se publican indicadores del capital intelectual. Choi et al. (2000) realizaron un estudio en Estados Unidos que muestra la relación entre los informes de los activos intangibles, los gastos de amortización asociados y el valor de mercado del patrimonio de las empresas. Los resultados indicaron que el mercado financiero valora positivamente los informes de los activos intangibles.

Patel y Pavitt (1995; sintetizado en Cañibano et al. (1999, pp. 8)) encontraron que la inversión en I \& D había aumentado en más de $100 \%$ entre los años 1967 y 1990 para los cinco mayores países de la OECD y para el caso de Japón el crecimiento fue de 900\%. Análisis basados en el Manual de Oslo (OECD (1992,1996); Comisión Europea (1996)) mostraron que la I \& D es 
sólo una de las actividades que se puede extraer en el marco de la innovación; existen otras variables como adquisición de tecnología, marketing, desarrollo de software, entrenamiento, diseño, etc., y todas las actividades que permiten la implementación de nuevas tecnologías o desarrollos de nuevos productos o procesos. Las inversiones en innovación son principalmente entendidas como adquisiciones de poder de futuros ingresos, y podría ser considerado como un activo susceptible a ser reconocido y revelado en los estados financieros.

Cañibano et al. $(1999$, p. 32) se refirió a diversos autores de la literatura que han estudiado la relevancia de la I \& D, tales como Mortensen et al. (1997) y Ben-Zion $(1978,1984)$. El primero argumentó que numerosos estudios habían documentado una relación estadísticamente significativa entre I \& D y el valor de mercado de la compañía, y que el impacto de I \& D sobre el desempeño de las compañías va a depender de cada país. El segundo encontró que la diferencia entre el valor de mercado y valor libro de las empresas está correlacionada con los gastos en Innovación y Desarrollo. Este resultado evidencia que los inversionistas valoran este tipo de inversiones, ya que afirman la posición competitiva de la compañía.

Asimismo, Cañibano et al. (1999) menciona a Grabowski y Mueller (1978) que encontraron que las firmas en industrias intensivas en investigación ganaban significativamente mayores retornos sobre su capital en I \& D, y Woolridge (1988) que demostró una reacción positiva de los inversionistas frente a anuncios de inversión en I \& D de las empresas, lo que implicaría que el mercado no es miope, y que las expectativas de gasto en I \& D contribuyen a aumentar los ingresos futuros. Hirschey (1982) también encontró que, en promedio, los gastos en publicidad y en I \& D tienen un efecto positivo y significativo en el valor de mercado. La validez de estos resultados son corroborados por una gran cantidad de autores, mencionados en el mismo paper, entre ellos Chan, Martín y Kensinger (1990), Connolly y Hirschey (1990), Morck y Yeung (1991) y Doukas y Switzer (1992).

En todos estos estudios se documentó una consistente reacción positiva del precio de la acción frente a anuncios de aumento en gastos en I \& D, incluso en presencia de declinación de los ingresos. Según Vicente (2000) los efectos de las inversiones en I \& D sobre los beneficios, son diferidos y con retardo variable. Por su parte, los efectos de las inversiones publicitarias constituyen activos "invisibles" que tienen efectos de largo plazo sobre los beneficios.

Sin embargo, un estudio de Chan et al., (1999) no soporta una relación directa entre los gastos de I \& D y los retornos futuros de-las acciones. Un alto nivel de gastos en I \& D, afectan las utilidades informadas en los estados financieros, sugiriendo que se pueden producir distorsiones al considerar más estos gastos, que la capitalización de los costos de I \& D, si los inversionistas fallan al no hacer estos ajustes y considerar los beneficios futuros de I \& D.

La realidad en Chile en el tema de Innovación y Desarrollo es muy distinta a la de otros países dado que existen bajísimas inversiones en este concepto, principalmente por condiciones estructurales de la economía.

Según el Global Competitiveness Report (2002,2003), Chile al año 2000 invertía el $0,56 \%$ del PIB en I \& D, cifra que corresponde a la investigación de empresas, universidades y gobierno. Esta cifra es bastante pequeña en comparación con países desarrollados como Corea $(3,31 \%)$ y Finlandia $(2,17 \%)$. 


\section{Estudio empírico}

El objetivo de este estudio es, tal como se indicó en la introducción, conocer la importancia que asignan los analistas financieros a los intangibles (factores no financieros) al evahuar las empresas, para ello se utilizó la siguiente metodología:

\subsection{Metodología}

El estudio se desarrolló en la ciudad de Santiago, donde están concentrados la mayoría de los analistas financieros del país, mediante encuestas a una muestra de analistas financieros. Sin embargo, previamente se hizo una investigación exploratoria, que consistió en entrevistas con siete agentes directamente relacionados con el tema (ejecutivos de Corredoras de Bolsas, analistas financieros de Administradoras de Fondos de Pensiones). De estas entrevistas se pudo esclarecer los principales factores que toman en consideración los analistas financieros en sus evaluaciones, ellos son: el ambiente económico, variables financieras y no financieras. Asimismo, fue posible apreciar que las variables no financieras sí se incluían en su análisis en forma explícita con alguna ponderación o bien implícitamente, modificando la tasa de descuento de los flujos de caja futuros esperados, a fin de calcular el valor de la empresa. ${ }^{4}$

Las principales variables no financieras reconocidas para valorar una empresa fueron:

- Calidad de la gerencia.

- Publicidad.

- Ética gerencial.

- Relación con proveedores.

- Regulación.

- Fidelización de clientes.

- Tecnología.

- Confianza en la información de la empresa.

- Imagen corporativa.

La entrevista en profundidad anteriormente descrita, dio indicios valiosos para construir la encuesta final de ocho preguntas (cerradas y abiertas).

La muestra del estudio esta compuesta por 32 Corredores de Bolsa, los cuales representan un 76,1\% del total de corredores ubicados en Santiago, cuyos datos fueron encontrados en un listado oficial de los Corredores de Bolsa de Comercio de Santiago y en guías comerciales.

Las encuestas fueron personalmente administradas y guiadas para disminuir el error de respuesta y no respuesta. Para procesar las preguntas cerradas de la encuesta se utilizó el software SPSS versión 11.0 con el cual se obtuvo valiosa información a través de análisis de frecuencias y análisis factorial.

4 Uno de los modelos de valorización de empresas más señalados en los textos de finanzas es: descontar los flujos de caja que se espera genere la empresa en el futuro, con una tasa de costo de capital pertinente al riesgo de esos flujos de caja. 
El cuestionario incluyo 15 variables no financieras las que deberían ser clasificadas por los encuestados, según grados de importancia en sus análisis de recomendación de compra de acciones, dando también la posibilidad de agregar otras variables no indicadas específicamente.

Con el objeto de reducir el número de esas variables a un grupo de factores subyacentes que las liagan más interpretables, se realizó un Análisis Factorial, previo comprobar que los datos eran compatibles con dicha herramienta estadística, para ello se utilizaron las pruebas $\mathrm{KMO}^{5}$ y de esfericidad de Bartlett ${ }^{6}$ (los resultados generaron un valor de 0.802 para la primera y de 0.0 para la segunda). Así mismo, para obtener los resultados adecuados a los datos, se utilizó la técnica de análisis de componentes principales, ${ }^{7}$ que toma en cuenta la varianza total de los datos. Al utilizar los métodos de extracción, encontramos cuatro factores relevantes, ${ }^{8}$ los cuales explican el $64.6 \%$ de la varianza total de las variables y que se detallaran posteriormente.

Los factores subyacentes obtenidos, fueron posteriormente incluidos en las consideraciones que hicieron los analistas financieros de la importancia que asignaban a los factores no financieros en sus análisis a empresas en distintos sectores económicos.

\subsection{Resultados}

Los analistas financieros fueron muy categóricos en responder que los aspectos no financieros son considerados en sus análisis; el 100\% de los encuestados respondió que sí usan información no financiera para de recomendar la compra de acciones.

\subsubsection{Ponderación de los aspectos no financieros en el análisis de una empresa}

Al analizar el grado de importancia que asignan los analistas a las variables no financieras en la recomendación de acciones (ver Gráfico 1) se puede apreciar que del total de la muestra, el $83.8 \%$ afirma que al menos el $20 \%$ de sus análisis están basados en aspectos no financieros. Cabe destacar que el 25.8\% de los encuestados señala que la ponderación de los aspectos no financieros está entre $20 \%$ y $30 \%$, seguido por el $19.35 \%$ de los encuestados que afirman que los aspectos no financieros representan entre $40 \%$ a $50 \%$ en su análisis para recomendar acciones y, aún hay algunos analistas que asignan mayores proporciones a los factores no financieros en sus análisis.

5 Kaiser Meyer Olking: Si el valor calculado es mayor que 0.5 podemos decir que la muestra se adecua para hacer análisis factorial.

6 La prueba de esfericidad de Bartlett prueba la hipótesis nula que dice que las variables no están correlacionadas en la población, si el p-value es menor a 0.5 quiere decir que la prueba está correcta.

7 Para mayor profundización sobre el tema, revisar Kinnear, T. y J. Taylor (1998). "Investigación de mercados".

8 Se incluyeron los factores con varianza mayor a 1.0 y se utilizó la matriz factorial rotada. 
Gráfico 1. Ponderación de los aspectos no financieros en la recomendación de compra de acciones

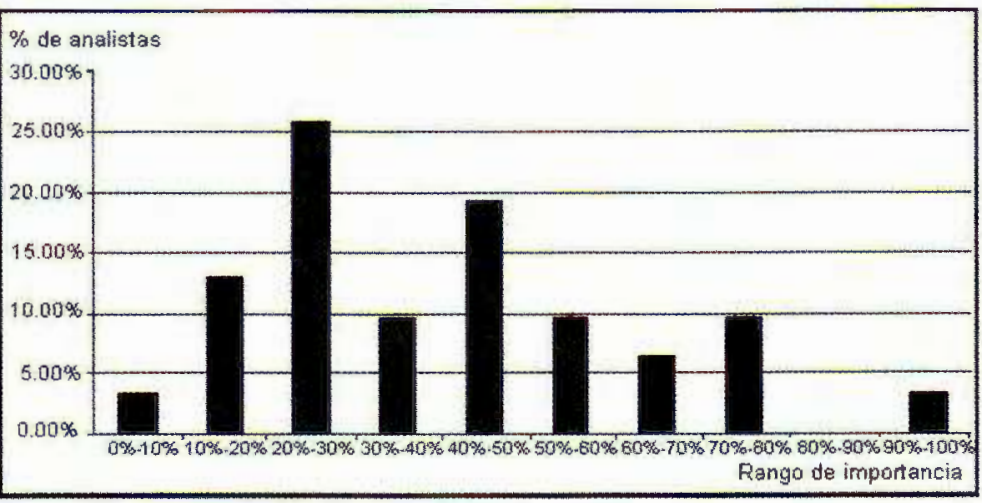

Fuente: Elaboración propia.

\subsubsection{Variables no financieras más importantes}

De acuerdo a los resultados obtenidos, las variables no financiera consideradas más importantes son "planes de expansión", "know how" 9 y "gestión corporativa" (ver Gráfico 2).

Los resultados son categóricos al indicar que los "planes de expansión" representan una variable fundamental para los analistas financieros al momento de analizar una empresa. En los últimos años, se ha observado el auge de inversiones y fusiones, a fin de expandir el área de negocios de las empresas y tener una mayor porción del mercado, este es el caso de supermercados, bancos, farmacias, tiendas por departamentos, entre otras. En un nivel de importancia similar, se indicaron las variables de "know how" (80.3\%) y "gestión corporativa" $(79.8 \%)$.

Otro grupo de factores no financieros señalados como importantes o muy importantes al momento de evaluar una empresa fueron: "credibilidad de la administración" (68.6\%); "ética gerencial" (66.7\%); "participación de mercado" (65.9\%). Solo un $13.8 \%$ considera esta última variable como poco o nada importante. Asimismo se indicaron como importantes o muy importantes la "infraestructura" que posee una empresa (61.8\%) y la "retención de clientes" $(60.4 \%)$.

Los tres elementos considerados en menor grado, como importantes o muy importantes fueron: "calidad de los recursos humanos" (52.1\%), "investigación, desarrollo e innovación" (48.4\%) y "habilidad para atraer y retener personas talentosas" (30.9\%). La innovación es fundamental para enfrentar la competencia y generar ventajas competitivas.

9 Con "Know how" nos referimos a toda la experiencia adquirida y desarrollada por la empresa para realizar sus actividades productivas. 
Gráfico 2. Variables no financieras más importantes para los analistas financieros

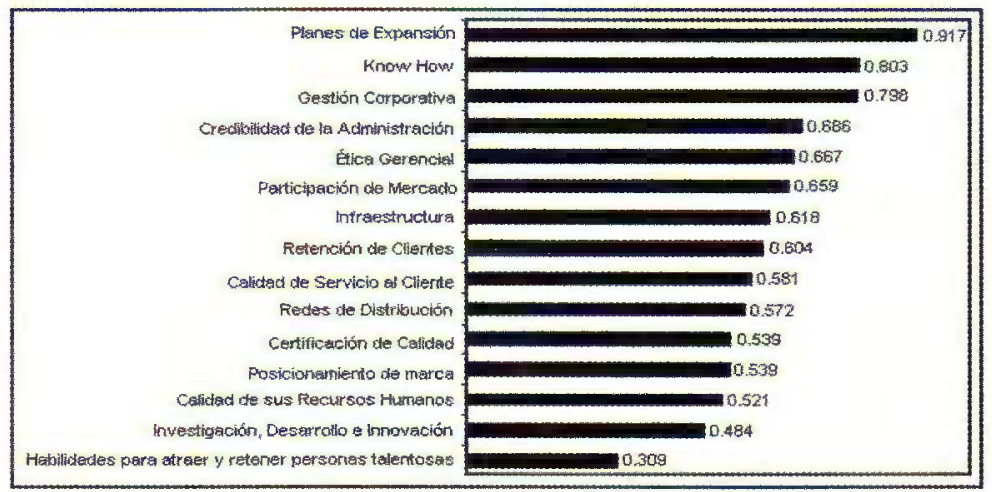

Fuente: Elaboración propia.

Al comparar los resultados anteriores con los que obtuvieron estudios en otros países como Inglaterra., Estados Unidos, Australia y Suiza, se pueden encontrar algunas similitudes: las variables no financieras que ocupan el primer y segundo lugar en Estados Unidos (Blitz (1997)) e Inglaterra (Eales et.al. (2000)) son la "ejecución y la calidad de la estrategia corporativa". En el presente estudio se incorporó la variable "gestión corporativa" que podría reunir estas dos variables, y resultó ser la tercera más importante para los analistas en Chile.

Por otra parte, en Chile la "credibilidad de la administración" ocupa el cuarto lugar, al igual que en Inglaterra; en Estados Unidos esta variable ocupa el quinto lugar. La "participación de mercado" está en el sexto lugar de importancia en Chile y también en Estados Unidos; en Inglaterra está en tercer lugar, al igual que para el mercado australiano (Morris et al. (1998)) y el mercado suizo.

Asimismo, se observa que en Chile la "retención de clientes" está en el octavo lugar de importancia, al igual que en Estados Unidos (Eccles y Kahn (1998)) y en Suiza (Eccles y Weibel (2000)) se considera en el noveno lugar. En cambio, según el estudio de Blitz (1997), esta variable se sitúa en el cuarto lugar en Estados Unidos al igual que en Australia.

Es interesante lo que sucede con la variable relacionada con la "I \& D", ya que ocupa el primer lugar de importancia en Suiza, el tercer lugar en Estados Unidos, el cuarto lugar en Inglaterra y Australia (según los estudios mencionados) y en Chile no está dentro de las primeras 10 variables relevantes, esto se podría explicar debido a que en Chile se invierten pocos recursos en investigación y desarrollo (en Chile se invierte $0.56 \%$ del PIB en "I \& D", mientras que en países como Finlandia o Corea se invierten $3.31 \%$ y $2.7 \%$ respectivamente). ${ }^{10}$ Se observó los datos de "I \& D" publicados en las notas a los estados financieros de varias sociedades anónimas, coll el fin de cuantificar los montos invertidos en ese rubro, sin embargo no se logró el objetivo deseado, pues

10 The Global Competitiveness Report 2002-2003, www.weforum.org 
los montos reportados en este concepto son muy pequeños o simplemente no existen.

\subsubsection{Otras variables no financieras}

Hay otras variables no financieras señaladas por los analistas financieros que podrían ser importantes al momento de hacer una recomendación de compra, venta o mantención de acciones. Estas respuestas se han agrupado en siete tópicos:

- La industria.

- Los ejecutivos de la empresa.

- Grupo económico.

- Macroeconómicas.

- La historia y el compromiso de la empresa.

- Información emitida por la empresa.

- Otras.

En el primer grupo, una de las variables más nombradas es el "marco regulatorio" que tienen que enfrentar las empresas, debido a que los analistas ajustan los pronósticos de los ingresos futuros de la empresa según las nuevas regulaciones, pues ellas claramente afectan el valor de la empresa. Para ejemplificar, podríamos mencionar el caso de Telefónica, empresa que ofrece un servicio que por ley, es obligatoriamente regulado por mantener la competencia e impedir un monopolio. De esta manera, la empresa debe acatar los decretos tarifarios que le fija los precios y le impide las que tienen relación con el gr upo económico al que pertenece la empresa.

Asimismo, para los analistas es fundamental conocer el grupo económico o holding que está detrás de cada empresa. La percepción general es que la estructura de propiedad indica lo que podría ser la empresa en cuanto a sus políticas y su gestión, es por ello que se interesan en variables como: "la capacidad de la administración del grupo", "los conflictos de intereses de los controladores", "el nivel de probidad del grupo económico", "la estructura de propiedad", "el comportamiento del grupo frente a cambios en el precio de las acciones", "la calidad del directorio", etc.

Un tercer grupo identificado, tiene relación con la historia y el compromiso de la empresa a lo largo del tiempo, dentro de esta categoría algunos analistas mencionaron como factor importante "la trayectoria de la empresa en cuanto al cumplimiento de los objetivos" y "el compromiso que ha demostrado en este sentido". Asimismo, se refirieron a la importancia de la historia de la empresa en cuanto a su trayectoria en el rubro en el que se desempeña la empresa analizada, y su historial económico y financiero.

El cuarto grupo identificado, tiene relación con la información que emiten las empresas al mercado, algunos analistas consideran que una empresa que entrega buena información, es más confiable que una que no la difunde, debido a que mientras más información entregue, menor es el riesgo ya que se reducen las asimetrías de información entre la empresa y los agentes del mercado. Se puede decir con esto que en Chile, los analistas perciben la importancia de los informes de las empresas al igual que en países como Inglaterra, Estados 
Unidos, Dinamarca, etc.; donde este tema es centro de discusión y de mucha investigación.

Algunos analistas consideran también ciertas variables relacionadas con los ejecutivos de la empresa, en este grupo se mencionó la antigüedad de los ejecutivos (o años de servicio), sus estudios (años de estudio, si realizó algún postgrado o un MBA). Esta información la obtienen principalmente mediante conversaciones con personas pertenecientes a la empresa o que tienen relación con ella.

Un sexto grupo, incorpora las variables macroeconómicas. Si bien el impacto de estas variables es muy importante para el análisis del valor de la empresa, este estudio no las incluye dentro de los activos intangibles, ya que no son variables internas a la organización y pertenecen al medioambiente, por lo tanto, afectan a todas las empresas con distinta intensidad según el sector.

- El escenario político y social del país.

- La situación económicas local y mundial.

- El clima macroeconómico interno.

- El crecimiento del PIB.

- El precio de algunos commodities, por ejemplo: petróleo, cobre, etc.

- Las monedas internacionales.

- La evolución de los países en donde opera la empresa.

Un último grupo reúne variables no financieras de distinta naturaleza, las cuales nombramos a continuación:

- Capacidad de asociación de las empresas con otros grupos o con la competencia

- Grado de integración del negocio.

- Pugnas por el control o grado de monopolio de la firma.

- Las perspectivas de crecimiento y desarrollo de la firma.

- Nuevos negocios.

- Productos ofrecidos.

- Posición conservadora o arriesgada frente a temas de riesgo en general.

- La reputación de la sociedad en que se desenvuelve la empresa.

- Capacidad comercial.

- Posicionamiento de la empresa en las carteras de los inversionistas. Clasi ficación de riesgo.

\subsubsection{Análisis factorial para variables no financieras}

Los resultados del análisis factorial empleado para reducir las 15 variables señaladas en la encuesta, a un número más reducido de factores, que facilitan el análisis, generó las agrupaciones de variables que se muestran en el Cuadro 1.

En la primera columna del Cuadro 1 se observan los factores que representan a un grupo de variables determinadas por el análisis factorial, en la 
columna de la derecha se observan el total de variables consideradas, agrupadas por factor.

El primer grupo de variables se denominó "fortaleza de la posición de mercado" ya que incorpora variables orientadas a la posición de la empresa con respecto a la competencia, como la participación de mercado, el posicionamiento de marca, retención del cliente y calidad del servicio al cliente. Pareciera ser que lo que caracteriza a este factor es la porción de mercado que posee la empresa, ya que incorpora variables relacionadas con la captación de clientes y además su retención. Este factor explica el $22 \%$ de la varianza total de las variables.

Al segundo grupo de variables se le llamó "fortaleza de la cultura corporativa", porque reúne los aspectos relacionados con la cultura de la organización, sus recursos humanos, su investigación, su innovación, y la ética gerencial. Este factor explica el $18 \%$ de la varianza total de las variables.

\section{Cuadro 1. Factores relevantes}

\begin{tabular}{|c|c|}
\hline Factores & Variables \\
\hline $\begin{array}{l}\text { Factor 1: Fortaleza de la posición } \\
\text { del mercado }\end{array}$ & $\begin{array}{l}\text { Posicionamiento de marca } \\
\text { Redes de distribución } \\
\text { Participación de mercado } \\
\text { Retención de clientes } \\
\text { Calidad de servicio al cliente }\end{array}$ \\
\hline $\begin{array}{c}\text { Factor 2: Fortaleza de la cultura } \\
\text { corporativa }\end{array}$ & $\begin{array}{c}\text { Habilidad para atraer y retener } \\
\text { personas talentosas } \\
\text { Ética gerencial } \\
\text { Investigación, desarrollo e innovación }\end{array}$ \\
\hline Factor 3: Experiencia en el rubro & $\begin{array}{c}\text { Certificación de calidad } \\
\text { Know How } \\
\text { Infraestructura } \\
\text { Planes de expansión }\end{array}$ \\
\hline $\begin{array}{c}\text { Factor 4: Calidad de la } \\
\text { administración }\end{array}$ & $\begin{array}{l}\text { Gestión corporativa } \\
\text { Credibilidad de la administración } \\
\text { Calidad de sus recursos humanos }\end{array}$ \\
\hline
\end{tabular}

Fuente: Elaboración propia.

El tercer factor llamado "experiencia en el rubro" agrupa las variables que tienen relación con los años de permanencia de la empresa en el mercado, como por ejemplo el know how que se ha adquirido del negocio, si ha logrado certificaciones de calidad, y si posee infraestructura acorde al desarrollo de su negocio. Este factor explica el $15 \%$ de la varianza total de las variables.

El cuarto factor se denominó "calidad de la administración" debido a que reúne características relacionadas con el capital humano de la empresa, vale decir la gestión de los directivos, de los administradores y con la calidad de los recursos humanos de la empresa. Cabe destacar que además de las variables 
relacionadas con el desempeño de las personas al interior de la empresa, también se incorpora a este factor el aspecto relacionado con la credibilidad que el mercado tiene acerca de la administración de la organización. Este factor explica el $9 \%$ de la varianza total de las variables.

Al cruzar los factores antes indicados, con el sector económico al que pertenece la empresa que estudian los analistas financieros, se obtuvieron algunos mapas ${ }^{11}$ que muestran la importancia relativa de los factores, según la percepción de los analistas encuestados, en el análisis de las empresas pertenecientes a los distintos sectores económicos.

En el Gráfico 3 (Mapa 1), se observa claramente que los analistas financieros encuestados consideran que el grupo de variables denominado "Fortaleza de la posición de mercado" es más importante para los sectores Alimentos y Bebidas, y Comercio y Distribución; ya que son los que se sitúan más a la derecha. Asimismo, la ubicación de los sectores Bancario y Financiero, y Telecomunicaciones indican que las variables relacionadas con este factor son también importantes para ellos, pero en menor medida, pues se encuentran más a la izquierda de los sectores Alimentos y Bebidas, y Comercio y Distribución.

Así, al momento de analizar el valor de las empresas pertenecientes a los sectores antes mencionados, las variables no financieras que consideran más importantes los analistas son el posicionamiento de marca, redes de distribución, participación de mercado, retención de clientes y la calidad de servicio al cliente.

En las empresas de los sectores Eléctrico, Agropecuario y Forestal este factor no es tan importante para los analistas encuestados, ya que como se observa en el mapa los sectores se ubican a la izquierda de éste.

Gráfico 3. Mapa 1.

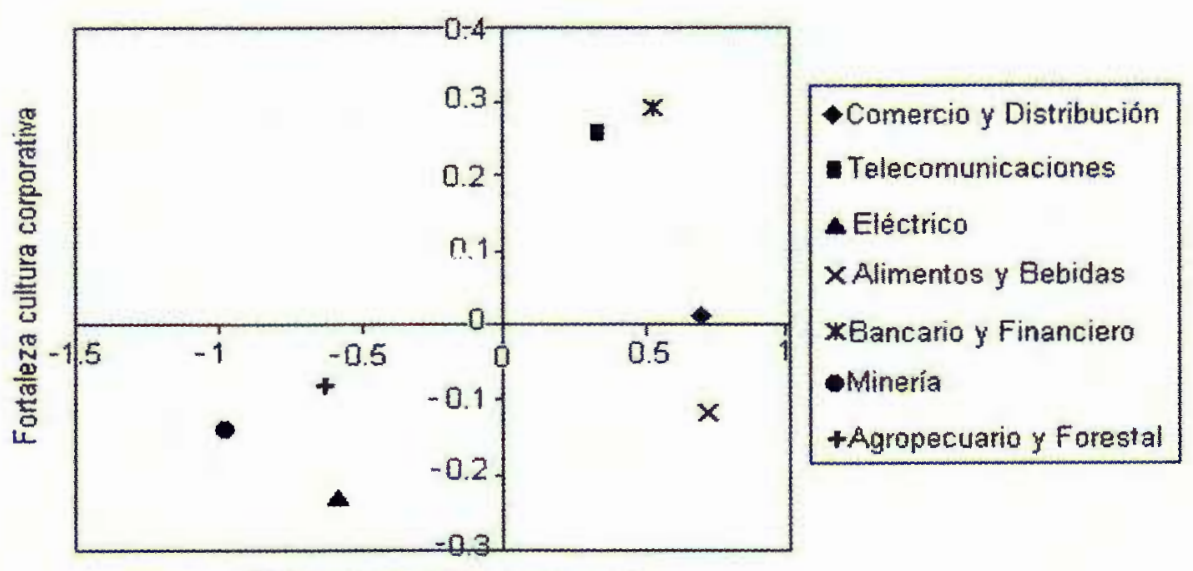

Fortaleza posición de mercado

Ante estos resultados, es coherente pensar que los analistas toman en cuenta las variables no financieras relacionadas con la posición de mercado y mayor

11 Los mapas de posicionamiento están basados en los datos de la matriz de factores. 
retención de clientes, especialmente en las empresas pertenecientes a sectores de servicios, cuyo objetivo, en parte, es tener una mayor participación de mercado. Tal es el caso del sector de Telecomunicaciones, donde la creciente rivalidad ha llevado a que las empresas compitan con estrategias más agresivas para obtener un mayor número de clientes. Lo mismo sucede en el sector Bancario, cuya tendencia actual es el de crecer a través de fusiones para ganar mayor porcentaje.

En cambio para los sectores primarios como el de Minería, Agropecuario y Forestal, y Sector Eléctrico no es tan importante para los analistas la cuota de mercado que poseen las empresas ya que tienen una cartera de clientes fija y con una rivalidad entre competidores baja.

De este mapa también se puede inferir la importancia del factor "Fortaleza cultura corporativa" en el análisis de empresas que pertenecen a los distintos sectores mencionados. De acuerdo al mapa se observa que la Fortaleza en la cultura corporativa es, según la percepción de los encuestados, muy importante para el sector Bancario y Financiero, seguido por el sector Telecomunicaciones y es menos importante para el análisis de empresas que pertenezcan a los sectores Comercio y Distribución, Agropecuario y Forestal, Minero y Eléctrico, tal como se observa en el Gráfico 3.

Esto quiere decir que variables como: habilidad para atraer y retener personas talentosas, ética gerencial e investigación, desarrollo e innovación son muy importantes para los analistas al momento de evaluar empresas pertenecientes a los sectores Bancario y Financiero, y Telecomunicaciones.

El sector Telecomunicaciones se caracteriza por invertir montos razonables en Investigación, Desarrollo e Innovación, debido a los constantes cambios tecnológicos que debe enfrentar, Sin embargo, el sector Bancario y Financiero no se caracteriza por ser un sector que invierte en investigación, más bien, este sector se caracteriza por la habilidad para atraer y retener personas talentosas, ya que por ser un sector de servicio, el capital humano es fundamental. La variable no financiera "ética gerencial" debiera ser una variable primordial para el análisis de empresas pertenecientes a los sectores Telecomunicaciones y, Bancario y Financiero.

En el Gráfico 4 (Mapa 2), se observan los otros dos factores identificados, el eje horizontal corresponde al factor llamado "Experiencia en el Rubro" y el eje vertical al factor "Calidad de la Administración".

Según la percepción de los analistas financieros encuestados, el factor "Experiencia en el Rubro" es más importante que en el sector Minero, seguido por el sector Agropecuario y Forestal. Los sectores Telecomunicaciones y Eléctrico se encuentran en el tercer lugar de importancia. Por otro lado, este factor es menos relevante para los sectores Alimentos y Bebidas, Comercio y Distribución y Bancario y Financiero, tal como lo muestra el mapa.

Por lo tanto, para el análisis de las empresas de los sectores primarios (Minería, Agropecuario y Forestal, y Eléctrico) es muy valioso el know how que las empresas tienen en sus respectivos rubros, así como también es importante la infraestructura que han adquirido a través de los años. En cambio para los demás sectores, estas variables no ocupan un lugar fundamental dentro del estudio de los analistas. 
Gráfico 4. Mapa 2.

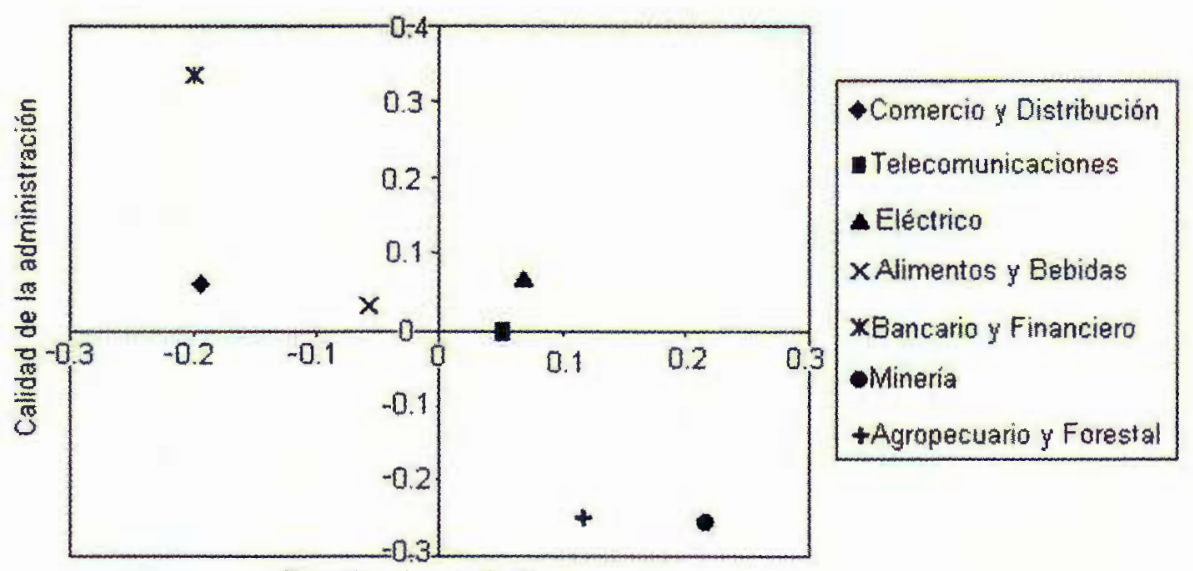

Experiencia en el rubro

Al último factor a analizar se le denominó "Calidad de la Administración", el cual como se dijo anteriormente, está compuesto por variables no financieras como la gestión corporativa, la credibilidad de la administración y la calidad de los recursos humanos.

Según la percepción de los analistas encuestados, este factor es muy apreciable para empresas del sector Bancario y Financiero. Para los sectores Comercio y Distribución, Alimentos y Bebidas y el de Telecomunicacionęs, este factor es relevante en mellor grado. Por último, para las empresas pertenecientes a los sectores Agropecuario y Forestal, y Minería, este factor no es tan relevante según los analistas encuestados.

Es destacable, la importancia que asignan los allalistas, a la gestión corporativa, la credibilidad de la administración y la calidad de los recursos humanos en el sector Bancario y Financiero. Esto quiere decir que los analistas toman en cuenta la administración de las empresas y el capital huniano en estos sectores, lo que es coherente si se piensa que son empresas de servicio.

Las conclusiones de estos mapas, son acorde a lo que se esperaba a priori que contestaran los encuestados. Así, las variables no financieras importantes para la empresa de cada sector son coherentes con el negocio de estos. Las demás combinaciones de factores en mapas de posicionamiento, conducen a resultados similares a los antes indicados.

Mediante los resultados obtenidos del análisis factorial, se construyó una matriz que relaciona el sector económico en estudio y los factores (Cuadro 2); cada cifra indica la importancia relativa de cada factor en el sector económico, así el número mayor (por sector económico) representa mayor importancia del factor ell el sector, mientras que el número menor representa al factor menos importante para el sector.

Como se aprecia en la primera fila, para el sector Comercio y Distribución, el factor más importante es el uno denominado "Fortaleza de la posición de 
mercado" (Factor 1), mientras que el menos importante de los cuatro factores, es el tres, "Experiencia en el rubro".

Para el sector Telecomunicaciones encontramos que el factor más importante es el uno, denominado "Fortaleza de la posición de mercado", mientras que el factor menos importante es el cuatro "Calidad de la administración".

Cuadro 2. Factores más importantes por sector

\begin{tabular}{|c|c|c|c|c|}
\hline Sector Económico & Factor 1 & Factor 2 & Factor 3 & Factor 4 \\
\hline Comercio y Distribución & 0.68493 & 0.0117 & -0.19661 & 0.06238 \\
\hline Telecomunicaciones & 0.30248 & 0.2531 & 0.05247 & -0.00454 \\
\hline Eléctrico & -0.58625 & -0.2272 & 0.06793 & 0.06378 \\
\hline Alimentos y Bebidas & 0.71924 & -0.1164 & -0.05848 & 0.03350 \\
\hline Bancario y Financiero & 0.48793 & 0.2987 & -0.20074 & 0.34164 \\
\hline Minería & -0.97517 & -0.1403 & 0.21713 & -0.25035 \\
\hline Agropecuario y Forestal & -0.63316 & -0.0797 & 0.11830 & -0.24641 \\
\hline
\end{tabular}

Fuente: Elaboración Propia.

Para el sector Eléctrico podemos apreciar que el factor más importante es el tres "Experiencia en el rubro", mientras que el menos importante es el uno. En el sector Alimentos y Bebidas tenemos que el factor más importante es el uno, "Fortaleza en la posición de mercado", mientras que el menos importante es el dos "Fortaleza de la cultura corporativa".

Para el sector Bancario y Financiero encontramos que el factor más importante es el uno "Fortaleza en la posición de mercado", mientras que el factor menos importante es el tres "Experiencia en el rubro". En el sector Minería podemos apreciar que el factor tres es el más importante "Experiencia en el rubro", mientras que el factor uno es el menos importante "Fortaleza de la posición de mercado". El sector Agropecuario y Forestal tiene como factor más importante al tres "Experiencia en el rubro", el menos importante es el uno "Fortaleza a la posición de mercado".

Así vemos que el factor que resulta más importante para un mayor número de sectores es el uno "Fortaleza de la posición de mercado", lo que demuestra su relevancia que es para los sectores Comercio y Distribución, Telecomunicaciones, Alimentos y Bebidas, Bancario y Financiero, lo que pareciera natural pues son sectores que necesitan estar en la parte final de la cadena productiva. Mientras que sectores como la Minería o Agropecuario y Forestal están más cerca de la primera parte de la cadena productiva, donde la experiencia en el rubro es fundamental.

\subsubsection{Análisis de frecuencia por sector}

Para complementar el análisis factorial, se estudia cada variable no financiera para cada sector económico, el Cuadro 3 muestra las frecuencias de respuestas 
"importante" y "muy importante" que los analistas financieros asignaron a esas variables. ${ }^{12}$

Los resultados indican:

- Para el sector Comercio y Distribución, las variables no financieras más relevantes son los "planes de expansión" (87.5\%), "posicionamiento de la marca" $(81.3 \%)$, "redes de distribución" $(81.2 \%)$, lo que concuerda con las principales ventajas competitivas que debería tener una empresa orientada a ese ruoro.

- Para el sector Telecomunicaciones, la variable no financiera más relevante es "planes de expansión" con (96.9\%), hecho que refleja la importancia para las empresas de Telecomunicaciones de expandir sus redes de comunicaciones, a fin de obtener una masa crítica de clientes que les permita aprovechar sus economías de escala. Las otras dos variables que le siguen en importancia a este sector son "know how" (81.3\%) y "retención de clientes" (78.2\%).

- Para el sector Eléctrico, los elementos más importantes son los "planes de expansión" (93.8\%), "know how" (75.0\%) y "gestión corporativa" (71.9\%).

Cuadro 3. Importancia de las variables no financieras por sector.

\begin{tabular}{|c|c|c|c|c|}
\hline & $\begin{array}{c}\text { Comercio y } \\
\text { distribución }\end{array}$ & $\begin{array}{c}\text { Telecomu- } \\
\text { nicaciones }\end{array}$ & Eléctrico & $\begin{array}{c}\text { Alimentos } \\
\text { y bebidas }\end{array}$ \\
\hline $\begin{array}{c}\text { Gestión corporativa } \\
\text { administración }\end{array}$ & $71.9 \%$ & $75.0 \%$ & $71.9 \%$ & $81.3 \%$ \\
\hline $\begin{array}{c}\text { Redes de } \\
\text { distribución }\end{array}$ & $75.0 \%$ & $65.7 \%$ & $65.6 \%$ & $56.2 \%$ \\
\hline $\begin{array}{c}\text { Know how } \\
\text { Participación de } \\
\text { mercado }\end{array}$ & $75.0 \%$ & $81.3 \%$ & $75.0 \%$ & $65.6 \%$ \\
\hline $\begin{array}{c}\text { Posicionamiento de } \\
\text { marca }\end{array}$ & $81.3 \%$ & $78.1 \%$ & $56.2 \%$ & $78.2 \%$ \\
\hline $\begin{array}{c}\text { Retención de } \\
\text { clientes }\end{array}$ & $71.8 \%$ & $78.2 \%$ & $37.6 \%$ & $68.8 \%$ \\
\hline $\begin{array}{c}\text { Calidad de servicio } \\
\text { al cliente }\end{array}$ & $75.0 \%$ & $71.9 \%$ & $40.6 \%$ & $68.8 \%$ \\
\hline $\begin{array}{c}\text { Planes de } \\
\text { expansión }\end{array}$ & $87.5 \%$ & $96.9 \%$ & $93.8 \%$ & $90.7 \%$ \\
\hline
\end{tabular}

Fuente: Elaboración propia.

12 Para efectos de este análisis, se sumó los porcentajes de las respuestas "importantes" o "muy importante", sólo se consideraron aquellas variables que en algún concepto tenían un grado de "importante" o "muy importante" mayor a. 75\%, bajo este criterio quedaron fuera la variable "Certificación de calidad", "Habilidad para atraer y retener personas talentosas", "Calidad de recursos humanos", "Investigación, desarrollo e innovación", "Infraestructura" y "Ética gerencial". 
Tabla 3. (continuación)

\begin{tabular}{|c|c|c|c|}
\hline & $\begin{array}{c}\text { Bancario y } \\
\text { Financiero }\end{array}$ & Minería & $\begin{array}{c}\text { Agropecuario y } \\
\text { Forestal }\end{array}$ \\
\hline Gestión Corporativa & $81.3 \%$ & $59.4 \%$ & $65.7 \%$ \\
\hline $\begin{array}{c}\text { Credibilidad de la } \\
\text { administración }\end{array}$ & $81.3 \%$ & $59.4 \%$ & $62.5 \%$ \\
\hline $\begin{array}{c}\text { Redes de } \\
\text { distribución }\end{array}$ & $68.7 \%$ & $21.9 \%$ & $37.5 \%$ \\
\hline $\begin{array}{c}\text { Know how } \\
\text { Participación de } \\
\text { mercado }\end{array}$ & $68.8 \%$ & $68.7 \%$ & $68.8 \%$ \\
\hline $\begin{array}{c}\text { Posicionamiento de } \\
\text { marca }\end{array}$ & $71.9 \%$ & $15.6 \%$ & $50.0 \%$ \\
\hline $\begin{array}{c}\text { Retención de } \\
\text { clientes }\end{array}$ & $84.4 \%$ & $31.3 \%$ & $37.5 \%$ \\
\hline $\begin{array}{c}\text { Calidad de servicio } \\
\text { al cliente }\end{array}$ & $81.3 \%$ & $25.0 \%$ & $31.3 \%$ \\
\hline $\begin{array}{c}\text { Planes de } \\
\text { expansión }\end{array}$ & $81.3 \%$ & $81.3 \%$ & $90.6 \%$ \\
\hline \multicolumn{2}{|c|}{} & & \\
\hline
\end{tabular}

Fuente: Elaboración propia.

- Para el sector Alimentos y Bebidas, los factores más importante son los "planes de expansión" (90.7\%), "redes de distribución" (84.4\%) y "posicionamiento de la marca $(84.4 \%)$, variables que podemos agrupar según la extracción de factores dados por el análisis factorial en "Fortaleza de la posición de mercado", lo que denota la importancia de este factor en este sector económico.

- Para el sector Bancario y Financiero, el factor más relevante es "retención de clientes" (84.4\%) y luego "Gestión corporativa", "Credibilidad en la administración", "Calidad de servicio al cliente" y "planes de expansión", todas con $81.3 \%$, reflejando lo importante que es para este sector retener a los clientes y la cultura corporativa.

- Para la Minería, el factor más relevante es "planes de expansión" (81.3\%); con menor grado de importancia están: "know how" (68.7\%); "gestión corporativa" y "credibilidad de la administración", ambas con un nivel de frecuencias de $59.4 \%$.

- Para el sector Agropecuario y Forestal, las variables no financiera más importante son los "planes de expansión" (90.6\%), luego "know how" (68.8\%) y "gestión corporativa" (65.7\%), lo que indica la relevancia que se asigna a la administración en las empresas de este sector.

\subsubsection{Indicadores de las variables no financieras}

En relación a cómo miden los factores no financieros, considerados en los estudios de las empresas, los analistas financieros, podemos señalar lo siguiente: 
- Planes de Expansión: El 22\% de los analistas que consideran importante esta variable, no tiene ningún indicador de medición; el resto, es decir el $78 \%$, lo mide a través de información revelada por la empresa, como por ejemplo: política de inversiones, capacidad instalada, proyectos futuros, presupuesto destinado a inversiones, porcentaje que representa la inversión en el patrimonio. Asimismo, esta variable es valorada en forma más subjetiva a través de la prensa mediante anuncios de la empresa, rumores, etc.

- Gestión Corporativa: De las personas que la consideran importante, el $26 \%$, no sabe como medirla y el resto la mide en forma subjetiva a través de la percepción de mercado, o de un criterio propio. Estas percepciones, según los encuestados, se pueden obtener mediante la transparencia en el manejo de dividendos, opiniones e información pública, cumplimiento de metas, capacidad para llevar adelante proyectos, adaptabilidad a los escenarios mediante una adecuada planificación financiera, etc.

- Ética Gerencial: El 85\% de los encuestados asegura que la ética gerencial es una variable importante, ya que no recomendarían la compra de acciones de una empresa en donde la gerencia se percibe como poco ética. Pero esta afirmación es ambigua, ya que si se pensara en una empresa de estas características, teniendo además en cuenta que ella podría ser muy rentable por medio de una subida en el precio de sus títulos o por medio de mejores dividendos, se analizaría más la situación colocando estas dos características en una balanza. Los analistas miden esta varíable mediante la percepción de mercado, la cual es formada a través de la historia de la gerencia, encuestas hacia los clientes, prensa, conocimiento personal, etc.

- Credibilidad de la Administración: De los encuestados que encontraron esta variable importante, el $29.6 \%$ no sabe como medirla, en cambio el $70.4 \%$ restante lo mide a través de la percepción de mercado, acompañado con un historial de la gestión del administrador en la empresa y si es posible su rendimiento en otras empresas. Similar a la variable anterior, esta percepción está alimentada por medio del cumplimiento de estrategias, opiniones e información pública, juicio personal, etc.

- Innovación: El 36.4\% no tiene un indicador de medición para esta variable, mientras que el resto lo valora a través de la generación de nuevos productos, variedad y novedad en productos, el plan de inversiones de la empresa, información obtenida en juntas de accionistas, encuestas, etc.

- Participación de Mercado: De los analistas encuestados que consideraron esta variable importante, el $22.7 \%$ no tiene un indicador de medición, el porcentaje restante lo evalúa a través de cifras de ventas que entrega la empresa, datos de la industria, informes o estudios publicados, encuestas, etc.

- Infraestructura: De los analistas que consideraron esta variable importante, el $28.5 \%$ no tiene un indicador de medición, en cambio los otros analistas la aprecian a través del monto de activos fijos e inversiones, la capacidad instalada de la empresa: plantas, redes, sucursales, etc. También, se busca información sobre los planes futuros de la empresa, los cuales son comprobados en algunos casos mediante visitas a la empresa.

- Posicionamiento de Marca: De los encuestados que consideran esta variable importante para analizar una empresa, el $43 \%$ no tiene un indicador de 
medición para ésta, el porcentaje restante la valora o mide a través de estudios de mercado, opinión propia, liquidez de sus instrumentos, posibilidades de lograr alianzas estratégicas atractivas, etc.

- Investigación y Desarrollo: De los analistas encuestados que consideran que esta variable es importante al momento de analizar una empresa, el $35 \%$ no sabe como medirlo; los demás analistas la aprecian a través de los nuevos productos que ha generado la empresa, recursos destinados a I \& D, comparando el porcentaje del patrimonio que constituye la inversión en I \& D entre las empresas de la industria, información obtenida en charlas, encuestas, prensa.

- Calidad del Servicio al Cliente: De los analistas encuestados, que consideran que esta variable es importante para analizar una empresa, el $37 \%$ no tiene un indicador para medirla, y el resto la valora en forma subjetiva a través de la percepción u opinión propia y, de los clientes, obtenida en estudios que se han hecho por medio de encuestas directas a los clientes. Se mide también a través de la fidelidad de clientes.

- Retención de Clientes: De los analistas que consideran que esta variable es importante, el $37 \%$ no tiene un indicador de medición, y el resto, lo mide por medio de la tasa de deserción de clientes, seguimiento de ventas, fidelización de clientes a través de tarjetas de crédito, opinión propia y percepción de mercado.

- Know How: De los analistas encuestados que consideran que el conocimiento que se tenga de una empresa del rubro es importante, el $37 \%$ no sabe o no tiene un indicador de medición; el resto, lo hace a través de la experiencia, capacidad de adelantarse a la competencia, años operando en la industria, análisis de situación objetiva, crecimiento potencial de la empresa, etc.

- Calidad de sus Recursos Humanos: De los analistas encuestados que considera esta variable como importante al momento de analizar una empresa, el $44.5 \%$ de ellos no sabe medirlo y por ende no tiene un indicador, en cambio los demás, lo valora a través de la percepción de mercado, la cual se obtiene por medio de hablar con los insiders de la empresa, a través de visitas a la empresa de modo de observar el clima de trabajo y a través de una opinión propia subjetiva. También se hace al observar algunas variables objetivas como la experiencia de las personas que trabajan en el área de recursos humanos.

- Redes de Distribución: De los analistas que consideran que esta variable es importante al analizar una empresa, el $29.4 \%$ no tiene un indicador de medición para esta variable, en cambio el $70.6 \%$ restante lo valora a través de la demanda, locales instalados, visitas, cantidad y calidad de canales de distribución, prensa, costo de distribución vs la competencia, balances y memorias.

- Certificación de Calidad: E1 50\% de los encuestados no sabe como medirla y no tiene ningún indicador de medición; el $50 \%$ restante la valora en su gran mayoría a través de los informes ISO. ${ }^{13}$

- Habilidad para Atraer y Retener Personas Talentosas: De los encuestados que consideran esta variable importante, el $78.6 \%$ no tiene un indicador de medición. El porcentaje restante lo hace por medio de una opinión propia, percepción de mercado e información pública.

13 Estos informes son normas internacionales de calidad que emplean algunas empresas. 
Como se puede apreciar, las variables no financieras descritas anteriormente y usadas por los analistas, son de difícil medición objetiva en el mercado chileno, debido principalmente a la dificultad para obtener datos más allá de los que están obligatoriamente publicados. Los principales indicadores mencionados se obtienen de informaciones solicitadas al departamento de relaciones con el inversionista y presentaciones públicas hechas por la gerencia.

\subsubsection{Medidas financieras usadas frecuentemente}

Las variables financieras son los principales elementos que consideran los analistas financieros en sus estudios para evaluar una empresa; los indicadores utilizados por los analistas financieros fueron bastante similares.

Los analistas valoran las empresas según el horizonte de inversión o desinversión que se tenga. Cuando ese horizonte es de corto plazo, algunos analistas usan herramientas para el análisis técnico con el fin de predecir los precios de los valores bursátiles,a partir de la evolución histórica de precios de esta empresa. Hay quienes, hacen un análisis fundamental, el que consiste en proyectar los flujos futuros y descontarlos a una tasa de costo de capital adecuada.

Asimismo, los analistas encuestados hacen un análisis complementario, comparando razones financieras de la empresa con las de mercado; las más utilizadas son las siguientes:

- $\mathrm{EV}^{14} / \mathrm{EVITDA}^{15}$

- Precio/Utilidad

- Valor empresa/EVITAD

- ROE

- Precio/EVITAD

- ROA

- Utilidad por acción

- Leverage

- Precio/Patrimonio

- Índices de Liquidez (liquidez corriente, razón ácida)

Otros aspectos financieros de interés señalados por los analistas encuestados fueron: utilidad proyectada, margen operacional, política de dividendos, flujos de caja, variabilidad y tendencia de flujos, inversiones, resultados de la empresa, cobertura de gastos financieros.

\section{Conclusiones}

Durante el último tiempo, nos hemos visto inmersos en la denominada nueva economía, donde la globalización, la innovación y la información han generado

14 "EV" proviene de la sigla en inglés "Equity value", que quiere decir valor económico del patrimonio.

15 Sigla en inglés que corresponde a las ganancias antes de intereses, impuestos, depreciación y amortización. 
un gran cambio, tanto en la organización interna de las empresas como en la importancia de los recursos que ellas emplean. Es así, que los intangibles ejercen un papel fundamental en la creación de ventajas competitivas sostenibles en las empresas, las cuales han tenido que adaptarse a estos cambios para poder trascender en el tiempo.

Ante este nuevo escenario han surgido nuevas necesidades de información que complementen los Estados Financieros, va que éstos no señalan todos los activos intangibles de las empresas; así lo han demostrado diferentes estudios realizados en distintos países como: Estados Unidos, Inglaterra, Australia y Suiza. En ellos se establece que los agentes del mercado de capitales demandan cada vez más información sobre los aspectos no financieros de las organizaciones. Este trabajo pretende dar a conocer lo que está ocurriendo en Chile en este ámbito, para lo cual se investigó la participación de los factores no financieros en la recomendación de compra de acciones, efectuada por analistas financieros de los Corredores de Bolsa.

Los resultados son categóricos al indicar que los analistas sí consideran los aspectos no financieros al momento de analizar una empresa; para el $80 \%$ de los analistas encuestados la ponderación de los aspectos no financieros en la recomendación de compra de acciones, representa más de un $20 \%$.

La variable no financiera más relevante para el análisis de las empresas pertenecientes a todos los sectores es "planes de expansión", lo que hace pensar que para los analistas son importantes las oportunidades de crecimiento, las proyecciones y las inversiones de las organizaciones. Otros factores señalados como importante son: el "know how", la "gestión corporativa", "credibilidad de la administración", "ética gerencial" y "participación de mercado".

Se pudo apreciar también, que la relevancia de las variables no financieras varía según el sector econónico. Es así, que variables como "gestión corporativa", "credibilidad de la administración" y "ética gerencial" son bastante consideradas por los analistas en sus análisis de empresas pertenecientes al sector Bancario y Financiero. Por su parte, "las redes de distribución", "el posicionamiento de marca" y "la participación de mercado" son importantes para empresas de los sectores Comercio y Distribución, y Alimentos y Bebidas. Luego, para los sectores primarios como el Agropecuario y Forestal, y el Eléctrico es más relevante el "know how" y la "infraestructura".

Los resultados de este estudio, señalan que los analistas financieros no poseen indicadores para medir los factores no financieros que ellos toman en cuenta y más bien, tratan de informarse a través de conversaciones, percepciones o a través de la prensa.

En este escenario, se puede concluir que las empresas debieran tomar conciencia de que la identificación, la gestión, la medición y la comunicación de los factores no financieros son elementos fundamentales para poder contribuir a su valor y lograr atraer nuevos accionistas. Asimismo, los organismos encargados de emitir las normas contables debieran enfrentarse al reto de proporcionar nuevos estilos de informes que incorporen los activos intangibles y, de esta manera, mejorar la comunicación de información entre la empresa, los analistas y los inversionistas. 


\section{References}

Amir, E. and B. Lev (1996). Value-relevance of Nonfinancial Information: The Wireless Communication Industry. Journal of Accounting and Economic, 22, pp. 3-30.

Anderson, R.H. and M.J.Epstein (1996). The Usefulness of Corporate Annual Reports to Shareholders in Australia, New Zealand, and the United States: An International Comparison. Studies in Managerial and Financial Accounting, 4.

Barlett, S.A. and R.A. Chandler (1997). The Corporate Report and the Private Shareholder: Lee and Tweedie Twenty Years On. British Accounting Review, 29, pp.245-261.

Beattie, V. (1999). Business Reporting: The Inevitable Change. The Institute of Chartered Accountants of Scotland.

Ben-Zion, U. (1978). The Investment Aspect of Non Production Expenditures: An Empirial Test. Journal of Economics and Business, pp. 224-229.

Ben-Zion, U. (1984). The R \& D Investment Decisión and its Relationship to the Firm's Market Value: Some Preliminary Results. In Z. Griliches (ed.) R \& D, Patents and Productivity, University of Chicago Press.

Blitz, A., T. Siesfeld, and P. Bierbusse (1997). Measures that Matter. The Ernst \& Young Center for Business Innovation (C.B.S.).

Brown, S., K. Lo, and T. Lys (1999). Use of in Accounting Research: Measuring changes in Value Relevance over the Last Four Decades. Journal of Accounting and Economics, 28(2), pp. 83-115.

Bukh, N., C. Nielsen, P. Gormsen, and J. Mouritsen (2003). Intellectual Capital Statements on their way to the Stock Exchange? A comparison of IPO Prospectuses and new Reporting Forms. Aarhus School of Business.

Cañibano, L., M. Garcia-Ayuso, and M.P. Sánchez (1999). The Value Relevance and Managerial Implications of Intangibles: A Litearture Review. Proyecto Meritum.

Coleman, J. and R. Eccles (1997). Pursuing values: Reporting Gaps in the United Kingdom. PricewaterhouseCoopers.

Connolly, R.A. and M. Hirschey (1990). Firm Size and R \& D effectiveness: A Value-Based Test. it Economic Letters, pp. 277-281.

Chan, L., J. Lakonishok, and T. Sougiannis (1999). The Stock Market Valuation of Research and Development Expenditures. Working paper 7223. National Bureau of Economic Research.

Chan, S., J. Martín, and J. Kensinger (1990). Corporate research and development expenditures and share value. Journal of Accounting and Economics, 26, pp. 255-276.

Choi, W., S. Kwon, and G. Lobo (2000). MArket Valuation of Intangible Assets. Journal of Business Research, 49, pp. 35-45.

Doukas, J. and L. Switzer (1992). The Stock Market's view of R \& D Spending and Market Concentration. Journal of Economics and Business, pp. 95-114.

Eales, J., R. Schosmann, T. Andrew, and I. Williams (2000). Measures that Matter U.K.: An outside in Perspective on Shareholder Value Recognition. Center for Business Innovation. Ernst \& Young LLP.

Eccles, R. and H. Kahn (1998). Pursuing values: The Information Reporting Gap in the U.S. Capital Markets. PricewaterhouseCoopers.

Eccles, R. and S. Mavrinac (1995). Improving the Corporate Disclosures Process. Sloan Management Review, pp. 11-25.

Eccles, R. and P. Weibel (1998). Pursuing Value: The Information Reporting Gap in the Swiss Capital Markets. PricewaterhouseCoopers.

Eccles, R., D. Phillips, and H. Richards (1998). Value Reporting. Reporting Gaps in the United Kingdom. The Chief Executive's Perspective. PricewaterhouseCoopers.

Eccles, R., R. H. Herz, E. Keegan, and D. Phillips (2001). The Value Reporting Revolution. Moving Beyond the Earning Game. New York: John Wiley \& Sons.

Garcia-Meca, L. and M. Conesa (2003). Capital intelectual y analistas financieros. III Workshop Investigación Empírica. Contabilidad Financiera. Universidad de Alicante, España. 
Gelb, D.S. (2002). Intangible Assets and Firms' Dissclosures: An Empricial Investigation. Journal of Business Finance and Accounting, 9(3,4), pp. 457-476.

Gleb, D.S. and P. Zarowin (2002). Corporate Disclosure Policy and the Informativeness of Stock Prices. Review of Accounting Studies, 7, pp. 33-52.

Grabowski, H. and D. Mueller (1978). Industrial Research and Development, Intagible Capital Stocks and Firm Profit Rates. Bell Joumal of Economics, 9, pp. 328-343.

Hirschey, M. (1982). Intangible Capital Aspects of Advertising and R \& D Expenditures. Journal of Industrial Economics, 30(4), pp. 375-390.

Lev, B. and P. Zarowin, (1999). The Boundaries of Financial Reporting and how to Extend them. Journal of Accounting Research, 37, pp. 353-385.

Mavrinac, S. and T. Boyle (1996). Sell-Side Análisis, Non-Financial Perfomance Evaluation \& The Accuracy of Short-Term Earnings Forecast. Working paper. The Ernst \& Young Centre for Business Innovation.

Meritum (2002). Directrices para la gestión y difusión de informaciónm sobre intangibles. En Cañibano, L., L. Sánchez, M. García-Ayuso y C. Chaminade (eds.). Fundación Airtel Móvil.

Morck, R. and B. Yeung (1991). Why Investors Value Multinationality. Journal of Business, pp. 165-187.

Morris, G., R. Eccles, and J. Falconer (1998), Pursuing Value: Reporting Gap in the Australian Capital Markets. PricewaterhouseCoopers.

Mortensen, J., C. Eustace, and K. Lannoo (1997). Intangibles in the European economy. Paper presented at the CEPS Workshop on Intangibles in the European Economy. Brussels.

Patel, P. and K. Pavitt (1995). Patterns of Technological Activity: Their Measurement and Interpretation. In Stoneman, P. (ed). Handbook of the Economics of Innovation and Technological Change. Oxford: Basil Blackwell.

Ross, J. (1998). Capital Intelectual. Trand Management/MD. Volumen 1/Edición Especial. Octubre, pp. $56-59$

Serrano, C. (2000). Ejemplos de informes de activos intangibles. http://www.ciberconta.unizar.es/LECCION/capint2/INICTOhtml

Sidney, D., C. Stickney, and R. Weil (1982). Financial Accounting: An Introduction to Concepts, Methods, and Uses. 3 edición. Chicago. Driden Press.

Sullivan, P. Jr and P. Sullivan (2000). Valuing Intangibles Companies: An intellectual Capital Approach. Journal of Intellectual Capital, 1(4), pp. 328-340.

Valenzuela, E. (2003). Non-financial Factors in the Stock Investment decisions: Institutional Investors. IBEA Congress.

Vicente, J. L. (2000). Inversión en intangibles y creación de valor en la industria manufacturera española. Universidad de Salamanca.

Woolridge, J. (1988). Competitive decline and Corporate Restructuring: Is a Myopic Stock Market to Blame? Journal of Applied Corporate Finance, 1, pp. 26-36. 